\title{
The Malay Version of the European Organization for Research and Treatment of Cancer Quality of Life Questionnaire (EORTC-QLQ C30): Reliability and Validity Study
}

Yusoff $\mathrm{N}^{\mathrm{a}}$, Low WYb and Yip $\mathrm{CH}^{\mathrm{c}}$

${ }^{a}$ Women's Health Development Unit, School of Medical Science, Health Campus, Universiti Sains Malaysia

${ }^{b}$ Medical Education and Research Development Unit,

c Department of Surgery, Faculty of Medicine, University of Malaya

\begin{abstract}
Introduction: The Malay Version of EORTC-QLQ C3O was validated among Malaysian women who had undergone breast cancer surgery. Materials and Methods: Test-retest evaluation (i.e. three weeks and ten weeks following surgery) was carried out to examine the validity and reliability of the scale. The Cronbach's alpha value was used to determine the internal consistency, meanwhile, test-retest Intraclass Correlation Coefficients (ICC) indicates the reliability of the scale. Effect Size Index and Mean Differences interpret the sensitivity of the scale. Discriminant validity was evaluated by comparing two groups i.e. women who had mastectomy and women who had lumpectomy. Results: Internal consistencies are acceptable for Global Health Status (0.91), Functional domains (ranging from 0.50-0.89) and Symptomatology domains (ranging from 0.75-0.99). Intraclass Correlation Coefficient (ICC) ranged from 0.05 to 0.99 for Global Health Status and Functional domains, and ranged from 0.13 to 1.00 for Symptomatology domains. Sensitivity of the scale was observed in nearly all of the domains. Conclusion: The Malay Version of the European Organization for Research and Treatment of Cancer Quality of Life Questionnaire (EORTC-QLQ C30) is a suitable tool to measure the quality of life of women with breast cancer.
\end{abstract}

KEYWORDS: Breast cancer, internal consistency, discriminant validity, EORTC malay version, intraclass correlation coefficient

\section{INTRODUCTION}

Quality of life of the individual with cancer is a multidimensional concept which is constructed and developed by at least four areas: psychological functioning, physical functioning, social functioning, and symptoms and side effects. ${ }^{1}$ The EORTC QLQ-C30 Version 3.0 is the most recent version and is developed to assess the quality of life of cancer patients (Quality of Life Unit, EORTC Data Centre, Brussel). It is a copyrighted instrument, which has been translated and validated into 49 languages and is used in more than 3000 studies worldwide. This instrument is provided with the collaboration between Quality of Life Group and Quality of Life Unit in Brussels, Belgium. It is designed to be cancer specific, multidimensional in structure, appropriate for self-administration, applicable across a range of cultural settings and suitable

Corresponding author;

Yusoff $N$

Women's Health Development Unit,

School of Medical Science,

Universiti Sains Malaysia,

16150 Kubang Kerian, Kelantan, Malaysia.

e-mail: kelkatu2310@yahoo.com for use with additional site- or treatment-specific modules. This EORTC QLQ-C30 Version 3.0 consists of three scales:

- $\quad$ Functional scales - Comprises of physical functioning, role functioning, cognitive functioning, emotional functioning and social functioning.

- Symptom scales - Comprises of fatigue, pain, nausea and vomiting, dyspnoea, loss of appetite, insomnia, constipation and diarrhoea and financial difficulties.

- $\quad$ Global health status/ QOL scale.

For functional scale and symptom scales, the options for the items are rated on a four-point scale ranging from "not at al" (score one) to "very much" (score four). Meanwhile, the options for the items in the Global health status are rated on a seven-point scale, ranging from "very poor" (score one) to "excellent" (score seven). All of the scales and single-item measures range in score from zero to 100 . Linear transformation is used to standardise the raw score.

A high scale score represents a higher response level. Thus, a high score for a functional scale represents a high / healthy level of functioning and a high score for the global health status represents a high quality of life. Meanwhile, a high level of symptomatology / problems is presented by a high score for a symptom 
scale / item. The reliability of most of the sub-scales have been established with Cronbach's alpha coefficient more than 0.7 , except for the role functioning. ${ }^{2}$ Validity of the scale was proven by the significant correlations of all interscale, indicating that the scales assessed the distinct components of the quality of life construct. ${ }^{2}$ The validity of the scale is also shown by the functional and symptom measures that discriminate clearly the patients differing in terms of clinical status as defined by the Eastern Cooperative Oncology Group performance status scale, weight loss and treatment toxicity. In addition, the validity of scale has been indicated by statistically significant changes, in the expected direction, in physical and role functioning, global quality of life, fatigue and nausea and vomiting for patients whose performance status had improved or worsened during treatment. ${ }^{2}$

\section{MATERIALS AND METHODS}

Women with breast cancer were recruited from three main hospitals located in the capital city of Malaysia, i.e. Kuala Lumpur. These hospitals are The University of Malaya Medical Centre (UMMC), The Kuala Lumpur General Hospital (KLGH) and The Hospital Universiti Kebangsaan Malaysia (HUKM), Kuala Lumpur. Ethical approval was obtained from these various institutions as well as from the Ministry of Health Malaysia. Selection of the respondents was based on the inclusion criteria as follows: new cases of breast cancer, had undergone breast cancer surgery, were planned for adjuvant chemotherapy and had no current major diseases or chronic psychiatric condition.

Back Translation technique was implemented in the translation process of the Treatment of Cancer Quality of Life Questionnaire (EORTC-QLQ C30) from its original version (English) into Malay version, based on the standard protocol of the European Organization for Research and Treatment of Cancer. ${ }^{3}$ In this procedure, two Malay native speakers who were fluent in English did the "Forward Translation" i.e. English to Malay language. Similarly, another two Malay native speakers were employed to carry out the "Backward Translation” i.e. Malay to English Language. Women's feedback and comments on the difficulties in understanding or ambiguous meaning of certain words or sentences were recorded. The backward translation was re-implemented for the controversial words or sentences reported. The Malay Version of EORTC-QLQ C30 were then pre-tested and finalized before it can be used for this study. The permission for using and translating the BR23 was obtained from the Quality of Life Unit, EORTC Data Centre, in Brussels.

The final Malay Version of EORTC-QLQ C30 was distributed to 68 women with breast cancer who match the study inclusion criteria. All these women were approached in the Clinical Oncology Clinics and their names were retrieved from the oncologist and breast surgeon (co-author). Prior to questionnaires distribution, written informed consent was obtained from the women with breast cancer. Ethical approval for the study was obtained from the University Malaya Medical Centre (UMMC) Ethic Committee, Kuala Lumpur, Malaysia, and Ministry of Health, Malaysia.

Two different phases of evaluation were undertaken for the purpose of reliability and validity analyses i.e. (1) approximately two to three weeks following surgery (prior-to chemotherapy) and (2) approximately ten weeks following surgery (during chemotherapy). The nature of the scale is self-administered, thus, the women answered the scale themselves in a private room provided at the clinic.

Socio-demographic data was gathered from these women, such as, age, ethnicity, education, occupation, monthly income and duration of marriage. Medical information such as type of surgery, time since diagnosis and stage of breast cancer were also obtained.

The Statistical Package of Social Science (SPSS) version 15.0 was utilized in data analyses. The internal consistency of the Malay Version of EORTC-QLQ C30 was assessed by calculating the Cronbach's alpha coefficient. ${ }^{4}$ Meanwhile, the test-retest reliability was assessed using the Intraclass Correlation Coefficient (ICC) which ranges from one (perfectly reliable) to zero. ${ }^{5}$ Sensitivity of the scale was determined by calculating the mean differences between the evaluation at phase one (three weeks following surgery) and phase two (ten weeks following surgery) of the study, by means of a paired t-test. The effect size of each domain of the Malay Version of EORTC-QLQ C30 was also reported. ${ }^{5}$ In addition, the ability of the scale to differentiate the quality of life between women who had undergone mastectomy and women who had undergone lumpectomy (termed as discriminant validity), was also presented by the result of independent t-test analysis.

\section{RESULTS}

Socio-medical data of the women with breast cancer is depicted in Table I. The mean age of the women was $46.91(s d \pm 7.65)$ years old. The majority of the women $(n=53)$ had undergone mastectomy $(77.9 \%)$, meanwhile 15 (22.1\%) of them had undergone lumpectomy. Majority of them were diagnosed with stage two of breast cancer $(54.4 \%, n=37)$, followed by stage three $(38.2 \% ; n=26)$ and stage one $(7.4 \%, n=5)$. The time of diagnosis to their participation in the study was a mean of $52.04(\mathrm{sd} \pm 2.47)$ days. Classification by menopausal status, majority of these women were pre-menopausal $(61.8 \%, n=42)$, followed by the postmenopausal $(30.9 \%, n=21)$ and the peri-menopausal $(7.4 \%, n=5)$ group. These women had at least a secondary education $(64.7 \%, n=44)$, with a household monthly income of at least RM3000 or USD854.94 $(80.9 \%, n=55)$. Most of the women were unemployed or housewives $(58.8 \%, \mathrm{n}=40)$.

The internal consistency, Intraclass Correlation Coefficient, sensitivity and discriminant validity of the 
scale were presented in Tables II, III and IV. For the internal consistencies of the scales (see Table II), Cronbach's alpha values ranged from 0.50 to 0.91 for the Global Health Status and Functional domains, and ranged from 0.75 to 0.99 for the Symptomatology domains. In the mean time, the test-retest Intraclass Correlation Coefficient (ICC) ranged from 0.05 to 0.99 for the Global Health Status and Functional domains, and ranged from 0.13 to 1.00 for the Symptomatology domains (Table II). Sensitivity of the scale (Table III), as interpreted by the mean differences and Effect Size Index, was observed in most of the domains such as Global Health Status, Role Functioning, Emotional Functioning, Social Functioning, Cognitive Functioning, Nausea and Vomiting, and Appetite Loss. In term of discriminant validity, the significant differences between women who had gone through mastectomy and women who had gone through lumpectomy, were not detected, as indicated by Table IV.

\section{DISCUSSION}

Most of domains of the Malay Version of the European Organization for Research and Treatment of Cancer Quality of Life Questionnaire (EORTC-QLQ C30) exhibited excellent internal consistencies. This can be seen in Global Health Status, Emotional Functioning, Social Functioning, Fatigue, Nausea and Vomiting, and Pain. This finding almost parallel to that of previous studies which indicated the Cronbach's alpha of more than 0.75 in almost all domains of the European Organization for Research and Treatment of Cancer Quality of Life Questionnaire (EORTC-QLQ C30) except for the Role Functioning. ${ }^{2}$

The Malay Version of European Organization for Research and Treatment of Cancer Quality of Life Questionnaire (EORTC-QLQ C30) showed a range of effect size, from trivial to moderate $(0.00$ to 0.35$)$. This fact explains the effect of treatment phases on women's quality of life which is associated with the side-effect of chemotherapy treatment for breast cancer. Variations in the sensitivity of the scale was perhaps due to the treatment situation measured prior-to and during chemotherapy phases, and not because of the low sensitivity of the scale to detect a change.

It was observed that the Malay Version of European Organization for Research and Treatment of Cancer Quality of Life Questionnaire (EORTC-QLQ C30) did not discriminate the level of life's quality between the groups of women who had undergone mastectomy and women who had undergone lumpectomy. This could be accepted as the women's quality of life such as their functioning and symptomatology might not be affected by the type of surgery (mastectomy vs lumpectomy), except other aspect of life's quality such as body image and sexual attractiveness. This was also proven by other studies. ${ }^{6-9}$

In conclusion, the Malay Version of European Organization for Research and Treatment of Cancer Quality of Life Questionnaire (EORTC-QLQ C30) is suitable to be applied for the Malaysian women with breast cancer.

\section{Acknowledgements}

Special acknowledgements are dedicated to the University of Malaya, Kuala Lumpur, Malaysia, for the financial support (Fundamental Grant: FP058/2005C) and to all the women with breast cancer who had willingly taken part in this study.

\section{REFERENCES}

1. Tchekmedyian NS, Hickman M, Siau J, Greco A, Aisner J. Treatment of cancer anorexia with megesterol acetate: Impact on quality of life. Oncology 1990; 4:185-92

2. Aaronson NK, Ahmedzai S, Bergman B, et al. The European Organization for Research and Treatment of Cancer QLQ-C30: a quality of life instrument for use in international clinical trials in oncology. J Natl Cancer Inst 1993; 85:365-76

3. Cull A, Springers M, Bjordal K, Aaronson N, West K, Bottomly A. European Organization for Research and Treatment of Cancer (EORTC) Quality of Life Group Translation Procedure, 2nd edn. Quality of Life Unit, EORTC Data Centre, Brussels. 2002

4. Cohen J. Statistical power analysis for the behavioral analysis. Academic Press: New York, 1977

5. Deyo RA, Dichr P, Patrick DL. Reproducibility and responsiveness of health status measures. Control Clin Trials 1991; 12:142-58

6. Bleiker EM, Pouwer F, van der Ploeg HM, Leer JW, Ader HJ. Psychological distress two years after diagnosis of breast cancer: frequency and prediction. Patient Educ Couns 2000; 40:209-17

7. Cohen L, Hack TF, de Moor C, Katz J, Goss PE. The effects of type of surgery and time on psychological adjustment in women after breast cancer treatment. Ann Surg Oncol 2000; 7:427-34

8. Ganz PA, Schag AC, Lee JJ, Polinsky ML, Tan SJ. Breast conservation versus mastectomy. Is there a difference in psychological adjustment or quality of life in the year after surgery? Cancer 1992; 69:1729-38

9. Hopwood P, Fletcher I, Lee A, Al-Ghazal S. A Body Image Scale for use with cancer patients. Eur J Cancer 2001; 37:189-97 
Table I. Bio/socio-demographic and Medical Characteristics of the Women with Breast Cancer $(\mathrm{N}=68)$

\begin{tabular}{|c|c|}
\hline Age (mean $\pm s d)$ & $46.91 \pm 7.65$ years \\
\hline \multicolumn{2}{|l|}{ Education Levels: } \\
\hline Primary school & $10(15 \%)$ \\
\hline Lower secondary & $20(29.4 \%)$ \\
\hline Upper secondary & 24 (35.3\%) \\
\hline Form 6/Diploma/Certificate & 13 (19.1\%) \\
\hline Tertiary & $1(1.5 \%)$ \\
\hline \multicolumn{2}{|l|}{ Household Monthly Income (RM3.80=USD1): } \\
\hline Less than RM1000 & $17(25.0 \%)$ \\
\hline RM1001 to RM3000 & 38 (55.9\%) \\
\hline RM3001 to RM5000 & $6(8.8 \%)$ \\
\hline More than RM5000 & $7(10.3 \%)$ \\
\hline \multicolumn{2}{|l|}{ Occupation: } \\
\hline Professionals & $7(10.3 \%)$ \\
\hline Technicians and associate professionals & $5(7.4 \%)$ \\
\hline Clerical workers & $11(16.2 \%)$ \\
\hline Service workers/shop market sales workers & $4(5.9 \%)$ \\
\hline Housewives & $40(58.8 \%)$ \\
\hline Pensioner & $1(1.5 \%)$ \\
\hline \multicolumn{2}{|l|}{ Types of Breast Cancer Surgery: } \\
\hline Mastectomy & 53 (77.9\%) \\
\hline Lumpectomy & 15 (22.1\%) \\
\hline \multicolumn{2}{|l|}{ Menopausal Status: } \\
\hline Pre-menopausal & $42(61.8 \%)$ \\
\hline Peri-menopausal & $5(7.4 \%)$ \\
\hline Post-menopausal & $21(30.9 \%)$ \\
\hline \multicolumn{2}{|l|}{ Stages of Breast Cancer: } \\
\hline Stage 1 & $5(7.4 \%)$ \\
\hline Stage 2a & $21(30.9 \%)$ \\
\hline Stage 2b & $16(23.5 \%)$ \\
\hline Stage 3a & $16(23.5 \%)$ \\
\hline Stage 3b & $7(10.3 \%)$ \\
\hline Stage 3c & $3(4.4 \%)$ \\
\hline \multicolumn{2}{|l|}{ Duration of Breast Cancer } \\
\hline (mean $\pm \mathrm{sd}$ ) (From diagnosis to their partici & $52.04 \pm 2.47$ days \\
\hline
\end{tabular}


Table II. Internal Consistencies and Test-retest Intraclass Correlation Coefficient (ICC) of the Malay Version of the European Organization for Research and Treatment of Cancer Quality of Life Questionnaire (EORTC-QLQ C30) $(\mathrm{N}=68)$

\begin{tabular}{llll} 
PHASE 1 & PHASE 2 & $\begin{array}{l}\text { Internal } \\
\text { Consistency } \\
\text { (Cronbach's } \\
\text { alpha) }\end{array}$ & ICC \\
Mean (SD) & Mean (SD) & Test-retest \\
\hline
\end{tabular}

Global Health Status and Functioning Domains:

\begin{tabular}{lllll} 
Global Health Status & $70.57( \pm 19.51)$ & $61.28( \pm 17.92)$ & 0.91 & 0.96 \\
Physical Functioning & $72.75( \pm 15.09)$ & $74.85( \pm 18.54)$ & 0.73 & 0.17 \\
Role functioning & $42.92( \pm 17.08)$ & $31.86( \pm 13.61)$ & 0.72 & 0.83 \\
Emotional functioning & $59.48( \pm 21.17)$ & $52.68( \pm 19.23)$ & 0.85 & 0.99 \\
Cognitive functioning & $80.94( \pm 21.63)$ & $81.01( \pm 21.15)$ & 0.5 & 0.98 \\
\hline Social functioning & $57.92 \pm(22.11)$ & $41.67( \pm 25.43)$ & 0.89 & 0.05 \\
Fatigue & $46.53( \pm 19.94)$ & $50.91( \pm 20.73)$ & 0.75 & 0.16 \\
Nausea and vomiting & $11.98( \pm 23.88)$ & $32.08( \pm 23.00)$ & 0.99 & 0.2 \\
Pain & $60.42( \pm 21.62)$ & $61.18( \pm 21.81)$ & 0.77 & 0.99 \\
Dyspnoea & $10.42( \pm 18.39)$ & $10.97( \pm 18.60)$ & $\mathrm{S}$ & 0.98 \\
Insomnia & $49.17( \pm 19.41)$ & $50.63( \pm 19.45)$ & $\mathrm{S}$ & 1 \\
\hline Appetite loss & $37.92( \pm 14.71)$ & $45.78( \pm 18.62)$ & $\mathrm{S}$ & 0.13 \\
Constipation & $14.17( \pm 24.95)$ & $14.14( \pm 24.75)$ & $\mathrm{S}$ & 1 \\
Diarrhoea & $9.6( \pm 21.60)$ & $9.70( \pm 21.71)$ & $\mathrm{S}$ & 1 \\
Financial difficulties & $50.63 \pm( \pm 22.10)$ & $51.48( \pm 24.84)$ & $\mathrm{S}$ & 0.13
\end{tabular}

Phase 1 = Two weeks following surgery; Phase 2 = Ten weeks following surgery $S$ = Single question

Table III. Sensitivity of the Malay Version of the European Organization for Research and Treatment of Cancer Quality of Life Questionnaire (EORTC-QLQ C30) (N=68)

$\begin{array}{lllll}\text { PHASE 1 } & \text { PHASE 2 } & \text { Mean } & \text { Effect } & \text { p-value } \\ \text { Mean (SD) } & \text { Mean (SD) } & \text { Differences } & \begin{array}{l}\text { Size } \\ \text { Index }\end{array} & \end{array}$

Global Health Status and Functioning Domains:

\begin{tabular}{llllll} 
Global Health Status & $70.57( \pm 19.51)$ & $61.28( \pm 17.92)$ & 9.33 & 0.26 & $\mathbf{p}<0.001$ \\
\hline Physical Functioning & $72.75( \pm 15.09)$ & $74.85( \pm 18.54)$ & 2.19 & 0.06 & NS \\
Role functioning & $42.92( \pm 17.08)$ & $31.86( \pm 13.61)$ & 10.7 & 0.32 & $\mathbf{p}<0.001$ \\
\hline Emotional functioning & $59.48( \pm 21.17)$ & $52.68( \pm 19.23)$ & 6.97 & 0.17 & $\mathbf{P}<0.001$ \\
Cognitive functioning & $80.94( \pm 21.63)$ & $81.01( \pm 21.15)$ & 0.1 & 0.02 & $\mathbf{p}<0.05$ \\
Social functioning & $57.92 \pm(22.11)$ & $41.67( \pm 25.43)$ & 14.18 & 0.27 & $\mathbf{p}<0.01$ \\
Fatigue & $46.53( \pm 19.94)$ & $50.91( \pm 20.73)$ & 0.33 & 0.01 & NS \\
Nausea and vorniting & $11.98( \pm 23.88)$ & $32.08( \pm 23.00)$ & 17.91 & 0.35 & $\mathbf{p}<0.001$ \\
Pain & $60.42( \pm 21.62)$ & $61.18( \pm 21.81)$ & 0.5 & 0.01 & NS \\
Dyspnoea & $10.42( \pm 18.39)$ & $10.97( \pm 18.60)$ & 0.5 & 0.01 & NS \\
Insomnia & $49.17( \pm 19.41)$ & $50.63( \pm 19.45)$ & 0 & 0 & NS \\
\hline Appetite loss & $37.92( \pm 14.71)$ & $45.78( \pm 18.62)$ & 6.47 & 0.18 & $\mathbf{p}<0.05$ \\
Constipation & $14.17( \pm 24.95)$ & $14.14( \pm 24.75)$ & 0 & 0 & NS \\
Diarrhoea & $9.6( \pm 21.60)$ & $9.70( \pm 21.71)$ & 0 & 0 & NS \\
Financial difficulties & $50.63 \pm( \pm 22.10)$ & $51.48( \pm 24.84)$ & 2.49 & 0.05 & NS
\end{tabular}


Phase 1 = Two weeks following surgery; Phase 2 = Ten weeks following surgery NS = Not Significant

Table IV. The Discriminant Validity of the Malay Version of the European Organization for Research and Treatment of Cancer Quality of Life Questionnaire (EORTC-QLQ C30

$\begin{array}{llll}\text { MASTECTOMY } & \text { LUMPECTOMY } & t & \text { p-value } \\ \operatorname{Mean}(\mathrm{SD}) & \operatorname{Mean}(\mathrm{SD}) & & \end{array}$

Global Health Status and Functioning Domains:

\begin{tabular}{|lllll|} 
Global Health Status & $70.12( \pm 18.89)$ & $68.33( \pm 16.73)$ & 0.33 & NS \\
\hline Physical Functioning & $71.82( \pm 16.88)$ & $75.11( \pm 15.63)$ & 0.68 & NS \\
Role functioning & $44.34( \pm 18.47)$ & $45.56( \pm 17.21)$ & 0.23 & NS \\
Emotional functioning & $60.53( \pm 19.00)$ & $54.44( \pm 27.25)$ & 0.99 & NS \\
Cognitive functioning & $82.39( \pm 18.89)$ & $74.44( \pm 27.36)$ & 1.3 & NS \\
\hline Social functioning & $56.92( \pm 24.77)$ & $53.33( \pm 22.00)$ & 0.51 & NS
\end{tabular}

Symptomatology Domains:

\begin{tabular}{lllll|} 
Fatigue & $49.27( \pm 18.81)$ & $45.93( \pm 27.50)$ & 0.55 & NS \\
\hline Nausea and vorniting & $9.43( \pm 20.80)$ & $22.22( \pm 37.10)$ & 1.73 & NS \\
Pain & $64.78( \pm 22.56)$ & $58.89( \pm 19.79)$ & 0.92 & NS \\
Dyspnoea & $11.95( \pm 20.77)$ & $6.67( \pm 13.80)$ & 0.93 & NS \\
Insomnia & $46.54( \pm 16.46)$ & $53.33( \pm 24.56)$ & 1.26 & NS \\
\hline Appetite loss & $38.99( \pm 40.00)$ & $15.66( \pm 18.68)$ & 0.21 & NS \\
Constipation & $12.58( \pm 22.89)$ & $13.33( \pm 24.56)$ & 0.11 & NS \\
Diarrhoea & $6.92( \pm 21.04)$ & $17.78( \pm 27.80)$ & 1.64 & NS \\
Financial difficulties & $52.20( \pm 24.90)$ & $46.67( \pm 16.90)$ & 0.81 & NS
\end{tabular}

NS = Not Significant 